\title{
¿Por qué si hay tanta violencia en el camino ellas siguen migrando? Antropología, violencia y migración*
}

\author{
Why are they still migrating if there is so much violence \\ along the migration routes? \\ Anthropology, violence, and migration
}

\author{
JOSELIN BARJA CORIA**
}

\begin{abstract}
This article aims to construct an anthropological route to explore migration in transit through the framing of violence and gender and the feminist category of lived experience. The analysis is situated in relation to a continuum of violence that poor migrant central American women face from early in their lives and not just when migrating. Arguments are supported by a multi-sited ethnography approach and the idea of violence as a constitutive life element producing oppressive relationships of women before, during, and after the migratory experience.
\end{abstract}

Key words: migration in transit, central American women, anthropology of violence, experience

\begin{abstract}
Resumen
Este artículo busca construir una ruta antropológica para explorar la migración en tránsito a través de la mirada de la violencia y el género, mediante la categoría feminista experiencia vivida. Se realiza un análisis en relación con un continuo de violencias que mujeres migrantes centroamericanas pobres enfrentan desde etapas tempranas de vida y no sólo al migrar. Se ofrecen argumentos para utilizar la etnografía multisituada como enfoque metodológico para el estudio de la migración en tránsito. Se examina la violencia como elemento constitutivo para producir relaciones de opresión con las mujeres antes, durante y después de que migran.

Palabras clave: migración en tránsito, mujeres centroamericanas, antropología de la violencia, experiencia
\end{abstract}

$\mathrm{E}$ n las últimas dos décadas la migración en tránsito centroamericana en México cobró un interés particular en las políticas públicas locales y en la investigación académica. Para las ciencias sociales, estudiar este fenómeno ha representado un reto, por ser un tema usualmente caracterizado por la fugacidad del objeto de estudio, por la apenas creciente presencia de investigaciones analíticas que incorporen una perspectiva de género (Pintin-Perez, Rojas Wiesner y Buyan, 2018; Willers, 2018; De la O y Camus, 2018) y por tratarse de un fenómeno complejo circunscrito en la violencia que plantea dilemas éticos y metodológicos sobre la relación con los participantes y respecto a la presencia misma de quien investiga dentro de espacios violentos.

En esta colaboración se busca construir una ruta antropológica que aborde las des planteadas y abone a la discusión contemporánea de la antropología de la violencia en el ámbito de las migraciones.

\footnotetext{
* Artículo recibido el 30/01/19 y aceptado el 20/06/19.

** Universidad Autónoma Metropolitana, Unidad Iztapalapa, Departamento de Antropología. Av. San Rafael Atlixco núm. 186, col. Vicentina, Iztapalapa, 09340, Ciudad de México <jbarjac@gmail.com>.
} 
El texto se organizó en cuatro momentos analíticos: a) una propuesta metodológica para estudiar la migración en tránsito; b) una reflexión de la violencia como elemento consustancial de las migraciones indocumentadas o llamadas en tránsito; c) una viñeta representativa de violencia sexual y de género en la vida de las mujeres migrantes; y d) reflexiones sobre los dilemas que el trabajo de campo en contextos de violencia nos plantea en la investigación social.

En las conclusiones se retoma la pregunta que intitula este artículo: ¿por qué si hay tanta violencia en el camino ellas siguen migrando? para traducir los hallazgos en una propuesta práctica que contribuya y sirva de puente entre la antropología y la producción de políticas públicas en el entorno migratorio.

\section{Una propuesta metodológica para estudiar la migración en tránsito}

En la investigación social sobre la migración en tránsito encontramos algunas tendencias predominantes. Por un lado, estudios enmarcados en la necropolítica, como una nueva forma de gubernamentalidad en la que los Estados producen sujetos desechables (Estévez, 2018; Castro, 2017; Varela Huerta, 2017) y, por el otro, análisis descriptivos sobre crisis humanitarias, políticas de seguridad global y violaciones a derechos humanos (García Aguilar y Villafuerte Solís, 2014; Gómez-Johnson, 2015; Paris-Pombo, 2016). Ambos aportes, sin duda necesarios, se han concentrado más en el examen del Estado como productor de vidas desechables, que en la propia experiencia y sentido de agencia de quienes migran. El análisis de la movilidad humana desde la experiencia vivida constituye una tercera vía que puede contribuir al diálogo crítico en los estudios sobre violencia y migraciones contemporáneos. En esta línea delinearé lo que propongo.

Para ello, me remitiré a la propuesta feminista, en concreto a las contribuciones de Teresa de Lauretis y bell hooks. La primera en su búsqueda por nombrar el conocimiento silenciado de las mujeres llamado coloquialmente instinto, que requiere otro nombre, pues se trata de un proceso de comprensión, autorrepresentación y definición del yo como mujer que dotaría de carácter genérico a la subjetividad. Este conocimiento genérico llamado experiencia es el que deviene personas con cualidades raciales y genéricas que produce relaciones sociales para colocar a las personas de un sexo en lugares de opresión por su cualidad de género. "El sujeto humano es masculino" diría De Lauretis (1984: 255). Para De Lauretis la experiencia es "el proceso por el cual se construye la subjetividad de todos los sujetos sociales. A través de ese proceso uno se coloca a sí mismo o se ve colocado en la realidad social y con ello percibe como algo subjetivo esas relaciones -materiales, económicas e interpersonales- que son de hecho sociales y en una perspectiva más amplia históricas" (1984: 253).

Por su parte, bell hooks (2004) hace explícita la interseccionalidad de la experiencia sugiriendo la conceptualización experiencia vivida. La autorrepresentación y definición del yo como mujer no será igual para mujeres blancas que para negras o de color. En la experiencia vivida bell hooks encuentra la posibilidad de señalar las posiciones de privilegio o desventaja que hay en la experiencia de las personas aun siendo parte de un mismo género. Las relaciones vividas materializan esa subjetividad diferencial clasista y sexista en la experiencia vivida.

La finalidad de este artículo es dar cuenta de la experiencia vivida de mujeres migrantes indocumentadas que, inmersas en contextos de violencia desde sus países de origen, deciden migrar a pesar de saber que las rutas de tránsito son un escenario potencial para enfrentar nuevos eventos de violencia de género.

La construcción heurística de una investigación que sitúa como objeto de estudio a las mujeres migrantes en tránsito se topa con varias disyuntivas que resolver, por ejemplo: si el contacto con los sujetos de investigación es efímero, ¿quién es nuestra sujeta o nuestro sujeto de estudio? Si ellas transitan por rutas diversificadas ¿cuál es el espacio de investigación? Si en la experiencia las personas muestran una movilidad inherentemente impredecible, en la que se incluyen múltiples acciones como cambios de planes sobre el país destino, o ausencia de una ruta clara hacia un país destino ¿qué es la migración en tránsito? y, finalmente, ¿qué tipo de aproximación podemos plantearnos, cuando nuestro objeto de estudio son las mujeres?

Esta contribución aborda el viaje de tránsito como uno o varios momentos de la vivencia de movilidad humana que no se limita a un movimiento entre un país origen y uno de destino, sino a la experiencia itinerante de ir de un lugar a otro, hasta lograr las condiciones mínimas necesarias para establecer algún tipo de anclaje con alguna territorialidad. Con ello buscamos abonar al reconocimiento de la migración en cuanto fenómeno complejo.

La intención de mantener el término migración en tránsito como categoría analítica responde al interés por mostrar que tanto esta como otras clasificaciones (emigración, inmigración y migración de retorno) forman parte de un ordenamiento político que opera para producir personas en posición de ventaja y otras 
en posición de desventaja. Sobre el derecho al libre tránsito, la Constitución Política de los Estados Unidos Mexicanos establece en su artículo 11 :

Toda persona tiene derecho para entrar en la República, salir de ella, viajar por su territorio y mudar de residencia, sin necesidad de carta de seguridad, pasaporte, salvoconducto u otros requisitos semejantes. El ejercicio de este derecho estará subordinado a las facultades de la autoridad judicial, en los casos de responsabilidad criminal o civil, y a las de la autoridad administrativa, por lo que toca a las limitaciones que impongan las leyes sobre emigración, inmigración y salubridad general de la República, o sobre extranjeros perniciosos residentes en el país.

Toda persona tiene derecho a buscar y recibir asilo. El reconocimiento de la condición de refugiado y el otorgamiento de asilo político, se realizarán de conformidad con los tratados internacionales. La ley regulará sus procedencias y excepciones. ${ }^{1}$

En la práctica, este derecho al libre tránsito consagrado en nuestra Constitución se inviste de políticas migratorias que establecen los límites de esa movilidad humana, clasificando sujetos que pueden ingresar o salir de los países de forma regular y sujetos que tienen una prohibición legal para hacerlo, a saber, personas de color de piel morena u oscura, provenientes de países pobres, que en los hechos corporizan a la llamada migración en tránsito, y habrán de utilizar rutas migratorias construidas en el ámbito de la ilegalidad, en las cuales expondrán su vida.

De ahí que las experiencias de las personas en migración en tránsito narradas en documentales, reportajes periodísticos, informes de derechos humanos y en la academia sean siempre vivencias circunscritas a la violencia, como resultado de un sistema económico global que deposita indistintamente en las personas la responsabilidad de proveerse medios de subsistencia para acceder a una vida digna y que, en el caso de mujeres, materializa las formas de control a través de sus cuerpos y su sexualidad.

Las reflexiones aquí propuestas surgen del trabajo de campo realizado en los últimos cinco años investigando la migración en tránsito en el contexto mexicano desde mi experiencia como estudiante de un programa doctoral y de la participación activa que manten- go desde tiempo atrás en espacios ciudadanos de construcción de políticas públicas sobre temas de migración. El material de campo utilizado para la viñeta que conforma la tercera sección corresponde a dos proyectos con mujeres migrantes en 2014 y $2016 .^{2}$

Por lo anterior, la propuesta analítica aterriza en una pregunta pragmática: ¿Por qué si hay tanta violencia en el camino ellas siguen migrando?

La respuesta a este interrogante busca que la investigación social sea fuente en la elaboración de programas de política pública que atienden problemáticas migrantes, mismas que con frecuencia omiten las vivencias e intereses de las mujeres que migran (en ocasiones por falta de inclusión, otras veces por acciones de carácter asistencialista que esencializan un estado de vulnerabilidad inherente asociado a la mujer que migra).

En el terreno metodológico, consideré oportuno tratar el tema de la fugacidad del sujeto de estudio recurriendo a la propuesta planteada por George Marcus sobre la etnografía multisituada. Se trata de una aproximación suficientemente flexible para explorar los dilemas metodológicos expuestos con anterioridad.

La perspectiva multisituada es para Marcus "una modalidad que investiga y construye etnográficamente los mundos de vida de varios sujetos situados y también construye etnográficamente aspectos del sistema en sí mismo" (2001: 112).

Para lograr su función, la etnografía multisituada diversifica sus formas de construir conocimiento empírico mediante el seguimiento de personas, objetos, tramas, historias de vida y metáforas, entre otros, con la finalidad de reconstruir el proceso social más allá de los lugares donde ocurre el desplazamiento, un tipo de etnografía móvil que se interesa por la circulación de objetos, identidades, mercancías y procesos culturales en marcos espaciotemporales más difusos que los convencionales (Ferrándiz, 2011: 206).

En la migración en tránsito hay una circulación permanente de personas, símbolos y procesos culturales. En términos generales, la adaptación de la etnografía multisituada al estudio de la migración en tránsito plantea la posibilidad de estudiar varios procesos: las formas de conexión de las mujeres migrantes en un mismo espacio, la interrelación entre distintos espacios etnografiados de las rutas de tránsito, y las variadas

1 Diario Oficial de la Federación, 9 de agosto de 2019.

2 La recolección de datos de 2014 se efectuó dentro de un albergue para mujeres migrantes como parte de un proyecto para establecer una ruta de acción y protección con mujeres víctimas de violencia sexual en su tránsito por México. El material de campo incluyó 22 entrevistas individuales recopiladas de agosto a diciembre. Los datos de 2016 se obtuvieron en tres albergues de la Ciudad de México como parte de un proyecto para identificar necesidades específicas de protección para mujeres migrantes indocumentadas. Se llevaron a cabo tres grupos focales de diez, doce y ocho mujeres, respectivamente. Todas las participantes tenían un rango de edad de 16 a 48 años y eran originarias de Honduras, Guatemala, Nicaragua y El Salvador. 
posiciones que una persona podría ocupar en un periodo definido dentro de su trayectoria de vida.

Contrario al uso común de entender la etnografía multisituada como una aproximación metodológica que recopila su corpus de información en diferentes sitios, en el caso analizado en la viñeta del tercer apartado de este artículo se utiliza otra de las riquezas de la propuesta de la etnografía multisituada: etnografíar una multiplicidad de localidades a partir de las historias de las personas. El trabajo se enfocó en la conexión de tramas afines de mujeres que han vivido experiencias de tránsito en rutas migratorias distintas y que en el momento del trabajo de campo coincidían en un espacio particular: la Ciudad de México.

Se eligieron las tramas como corpus analítico pues éstas expresan tanto el sentido que las personas dan a los paisajes o espacios en los que circulan como las fantasías, idealizaciones comunes y afectos que circundan el ambiente de dichos lugares interconectados en la experiencia de tránsito.

Entiendo espacio en su significado simbólico y material: espacio como uno de los marcos sociales que junto con tiempo y lenguaje construyen tramas de sentido que sitúan a los sujetos en el mundo (Mendoza, 2004), pero que necesariamente está configurado en la realidad física y que encuentra una trama de sentido en la experiencia vivida. Espacios que, en opinión de Lefebvre, están llenos de pasiones, acciones, imágenes y símbolos, y que tienen en su centro un núcleo o magma afectivo, y por tal razón no pueden someterse a las reglas de la coherencia (2013).

Este acercamiento ayuda a entender que el concepto espacio como marco analítico se conforma de las tensiones, negociaciones y relaciones de poder que se entretejen en las interacciones entre los diferentes actores que participan de las redes de interconexión de las migraciones globales, y que todas estas relaciones de poder son de carácter afectivo. En ellas, se disputa el poder, la soberanía, el control migratorio y las prácticas para evadir dichos controles produciendo experiencias vividas diversas.

Por ende, la afectividad juega un papel importante en la experiencia vivida y a su vez conforma una dimensión colectiva asociada al espacio producido por sus actoras. Los espacios conectados en las rutas migratorias están cargados de emociones colectivas que organizan muchas de las estrategias que las mujeres utilizan, por ejemplo, preferir ciertos medios de transporte como el autobús y no el tren, elegir horarios más adecuados para realizar el viaje (por lo común de día), zonas por donde andar y zonas que evitar, lugares de alojamiento medianamente seguros, discursos que adoptar en caso de ser detenidas en un retén migratorio o por un grupo de pandilleros; en conclusión, un conjunto de tácticas, estrategias y saberes que se gestan en el viaje y se ponen en práctica por el carácter emocional de lo vivido y por la afectividad que representan los lugares por donde ellas transitan.

La descripción de estos espacios construidos en la experiencia de las mujeres, no obstante, es una interpretación de quien investiga. Al respecto, Besserer utiliza el concepto dialógico topología-topografía. Topología se refiere a la interpretación de los sujetos que llevan a cabo una serie de acciones, "la representación de lugares que fueron expresados en una narrativa como lugares experimentados" (2004: 8), mientras que la topografía informa de la reorganización que la persona que investiga realiza sobre las topologías encontradas en las narrativas de los actores.

El análisis que se presenta es de carácter topográfico. Se busca topografiar los referentes materiales y simbólicos que hacen que la representación del viaje

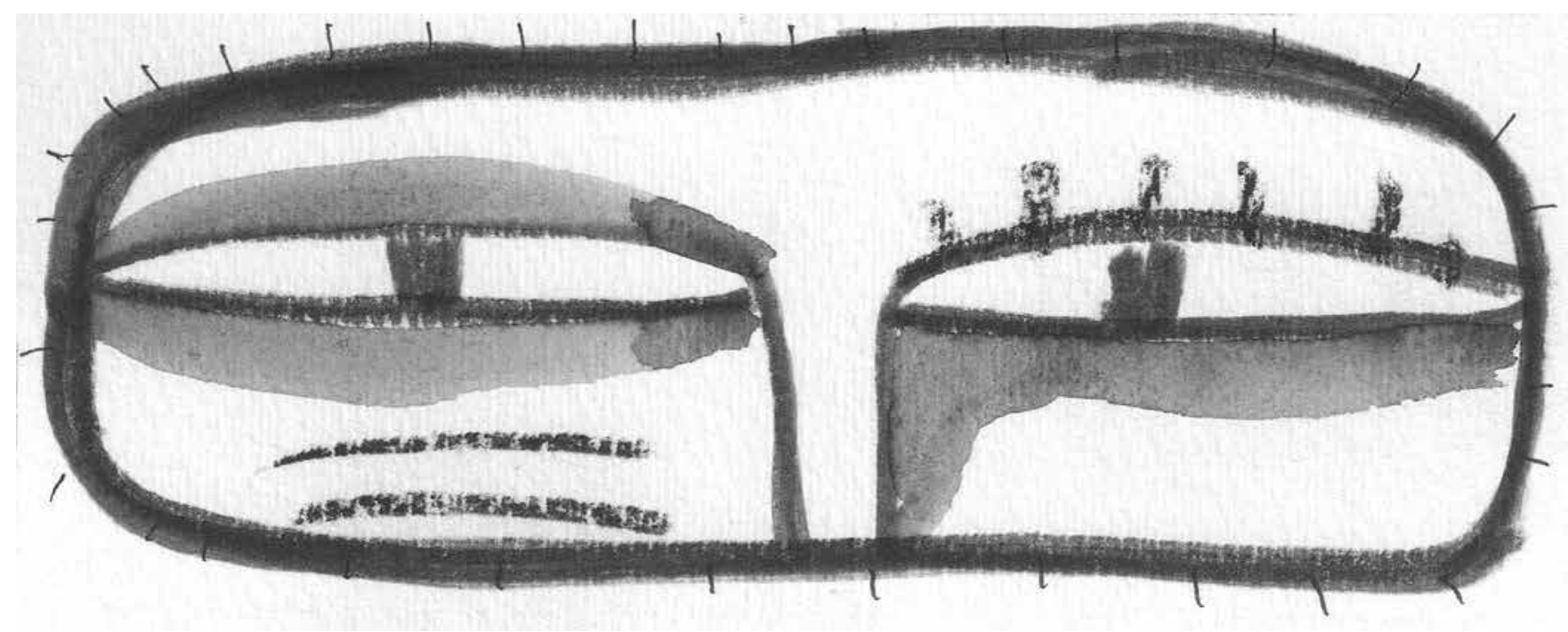


no sea igual para todas las mujeres, pese a transitar por los mismos lugares. Los referentes construidos en los lugares experimentados pueden marcar la diferencia entre vivir un viaje permeado por el terror del peregrinar en medio de un terreno simbólicamente cargado de muerte o un viaje sigiloso que, no obstante el conocimiento de las violencias circundantes, se distinga en su mayor parte por la esperanza.

Es también en las topografías donde encontramos el potencial de construir nuevas formas analíticas que den cuenta de que la geografía vivida por hombres y mujeres es genéricamente construida.

\section{La violencia como elemento consustancial de la migración en tránsito}

La migración en tránsito irregular desde el principio de este siglo dejó de caracterizarse por meras transacciones económicas entre contrabandistas, traficantes, prestadores de servicios del mercado negro de la industria migratoria, la complicidad de funcionarios corruptos y las redes de apoyo comunitarias para sostener los costos de un viaje con un origen y un destino.

La llamada ruta del terror o ruta del infierno migrante, como algunos medios de comunicación comenzaron a denominarla hace casi una década (NTR Periodismo crítico, 201 1; Zavaleta, 2013; Diario Imagen, 2018), se distingue peculiarmente por el sufrimiento, la crueldad y la muerte como referentes de la migración en espacios clandestinos. Testimonios como el siguiente, extraído del documental La Bestia, de Pedro Ultreras, son comunes en las representaciones mediáticas de la migración indocumentada: "En México hay caminos que no tienen regreso, no sabemos a dónde los deja el tren tirados, tal vez nuestra familia en nuestro país nos hace en EU, 'Que mi hijo o mi esposo ya no me ayuda, que se ha olvidado', y tal vez estamos sepultados en tierras mexicanas".

En contraste con la representación mediática de la migración en tránsito, la experiencia vivida da testimonio de un horizonte de vida que no se limita al cruce exitoso hacia los Estados Unidos o a las penurias del viaje. Por el contrario, se amplifica en un abanico de posibilidades que surgen de la incertidumbre en escenarios desconocidos en los que el destino de la vida misma es impreciso y está sujeto al dominio y voluntad de los grupos que controlan las rutas migratorias para llegar al norte de México, pero también de otros actores locales que proveen ayuda y protección, como los albergues y casas de migrantes. El capital de experiencia de vida previo de las personas que migran se sitúa dentro de estos marcos de riesgo y protección para activar distintas estrategias a fin de sortear las rutas de riesgo.

En el trabajo de campo un elemento común fue que las migrantes indocumentadas hacían énfasis en no tener prisa por continuar sus caminos. Se referían a sí mismas como distintas respecto de la experiencia de sus amigos, parejas, hermanos o conocidos hombres, afirmando que ellos lo querían hacer todo en un momento, que les urgía llegar y ya. Para ellas la posibilidad de seguir a Estados Unidos o quedarse en México era una pregunta de cada día, en ocasiones por considerar que en cualquier lugar podrían encontrar un trabajo para vivir mejor y lejos de las experiencias previas de violencia que las habían expulsado de su país -siendo las más frecuentes la violencia doméstica y las amenazas de pandillas centroamericanas por incorporarlas a la Mara S13 o a la Barrio 18 como parejas de alguno de los líderes-, o por haber experimentado violencia como "pago" para poder internarse en México-usualmente algún favor sexual o experiencias de asalto por delincuencia local o revisiones policiacas en las que se lleva a cabo la exposición y el tocamiento de sus cuerpos desnudos.

Pese a ello, las mujeres también habían puesto en práctica estrategias para contrarrestar esas violencias: hacerse amigas de algún miembro del grupo de guías o coyotes para protegerse en el resto del camino, utilizar el tren como último recurso de viaje y, de preferencia, trabajar por temporadas cortas para ahorrar y trasladarse en un medio más seguro totalmente aseadas y arregladas, con la intención de parecer turistas locales. ${ }^{3}$

Así, encontramos que hay una conformación espacial de la vivencia de tránsito circunscrita por distintas expresiones de violencia y a su vez hay acciones estratégicas de quienes migran para no someterse pasivamente a los mecanismos de control que pretenden sujetarles. Las estrategias son parte de un capital de saberes que la propia violencia ha producido en diversos momentos de sus vidas. Intentaré detallar a continuación.

La violencia no es un atributo específico de la vivencia del viaje de tránsito. Se trata de violencias múltiples que para ser entendidas deben ser exploradas en los contextos que las producen, en los entramados

3 En los operativos de revisión y verificación migratoria es sabido que los policías bajan a las personas de los autobuses por su "apariencia migrante", tomando como criterio estereotipos vinculados al color de piel, fenotipo de las personas y al arreglo personal. La mayoría de las veces, una persona poco aseada, de piel morena y con mochila será bajada de un autobús, aunque sea mexicana. 
de símbolos que las sustentan, en las significaciones que las personas adscriben a los lugares, en la manera en que perciben a los otros que perpetran la violencia y en cómo se perciben a sí mismas.

Estas topografías de las violencias son de interés colectivo porque explican las formas sistemáticas de reproducción de la violencia. Como afirma Blair, las geografías de las violencias "delimitan territorios, formas de ocupación y uso de ciertos espacios, los cuales hablan de una percepción generalizada de inseguridad y de miedo al tiempo que desaparece la confianza e incita a la búsqueda de espacios privados, se crean las condiciones para la producción y reproducción de la violencia" (2002: 61-62).

Lo que describiré en la siguiente viñeta surge de las entrevistas y grupos focales con mujeres de Centroamérica, y da cuenta de procesos transnacionales en los que criminalidad, migración y violencias se interconectan en diferentes espacios y ejercen una disputa en un territorio particular: los cuerpos sexualizados de personas construidas en código femenino (mujeres o mujeres trans).

\section{Violencia sexual y de género en la experiencia de las mujeres migrantes}

Mi inquietud por analizar el tema de la construcción genérica de la violencia en la migración en tránsito se origina a partir del contacto con mujeres en distintas localidades del país (Tabasco, Chiapas, Veracruz, Coahuila y Ciudad de México) durante el trabajo de campo de mi proyecto doctoral.

La contribución de este artículo se acota a los datos recopilados a partir de mi participación en dos proyectos orientados específicamente a mujeres migrantes. El primero, en 2014, cuando fui invitada a poner en práctica un programa piloto de atención a mujeres migrantes víctimas de violencia sexual durante su tránsito por México. El segundo, en 2016, que consistió en un diagnóstico de necesidades de mujeres y personas lésbico, gay, bisexual, transexual, transgénero, travesti e intersexual (LGBTTTI) migrantes y refugiadas en la Ciudad de México (Barja y Tlali, 2017). En este diagnóstico, la violencia sexual en sus variadas expresiones apareció de manera sistemática en la experiencia de vida de las personas y no sólo en el tránsito por México.

La Organización Mundial de la Salud define violencia sexual como "todo acto sexual, la tentativa de consumar un acto sexual, los comentarios o insinuaciones sexuales no deseados, o las acciones para comercializar o utilizar de cualquier otro modo la sexualidad de una persona mediante coacción por otra persona, independientemente de la relación de ésta con la víctima, en cualquier ámbito, incluidos el hogar y el lugar de trabajo" (2011, s/p).

En línea con esta definición genérica se ha documentado metódicamente que una proporción significativa de mujeres migrantes indocumentadas viven distintas formas de violencia en su camino hacia los Estados Unidos (Amnistía Internacional, 2010; Knippen, Boggs y Meyer, 2015; Redodem, 2015). Pero es poco común que estas contribuciones de denuncia social aborden las preguntas inevitables de por qué la pedagogía de la crueldad opera a través del ultraje de sus cuerpos y su sexualidad; y si el alcance de la definición arriba citada es suficiente para estudiar un fenómeno tan complejo.

Con pedagogía de la crueldad, Rita Segato (2013) ha introducido un tema imprescindible en el análisis de las prácticas de violencia hacia las mujeres, que en todo el planeta muestran una tendencia creciente y unas formas de ejercerse cada vez más recrudecidas. Segato asevera que mediante la ejemplaridad del castigo se logra mantener el control de un territorio. Se trata de una forma de enseñanza que mediante la ejemplaridad espectacular sobre la posesión del cuerpo de las mujeres tiene la intención de forjarlas como sujetos dóciles.

La violencia sexual en cuanto arma de guerra ha sido denunciada y documentada por especialistas en derecho internacional y humanitario y por agencias de ayuda humanitaria internacional cuando se enmarca en un contexto de conflicto armado, por ejemplo la Segunda Guerra Mundial, la Independencia de la India o de Pakistán, la creación de Bangladesh, los enfrentamientos étnico-religiosos en el Congo, el conflicto civil en Guatemala, por mencionar algunos.

En otros escenarios de conflicto no declarado o conflicto informal existe poco reconocimiento sobre los objetivos de la violencia sexual dirigida a los cuerpos feminizados. Atender esta inquietud implica, como primer paso, ampliar el espectro de análisis y el alcance de las definiciones de lo que entendemos por violencia sexual.

Históricamente ha sido un desafío contar con aproximaciones numéricas de la proporción de mujeres migrantes que en su paso por México, o en su establecimiento como trabajadoras indocumentadas, son víctimas de violencia sexual y de género.

Un informe de Amnistía Internacional aseguraba que seis de cada diez mujeres sufrían violencia sexual en su tránsito por México (2010: 5). En 2014, una investigación de Fusión afirmaba que, de acuerdo con 
entrevistas con encargados de casas de migrantes, cerca de 80 por ciento de mujeres migrantes estarían viviendo algún tipo de violencia sexual en México (Goldberg, 2014).

En el proyecto piloto de atención a mujeres migrantes víctimas de violencia en el que participé durante cinco meses en una casa para mujeres migrantes en la Ciudad de México estuve en contacto con 22 mujeres, de las cuales sólo una no expresó haber experimentado algún tipo de violencia de género en su vida. Aunque la entrevista tenía el objetivo de identificar violencia sexual en el viaje de tránsito, se encontró más de una expresión de violencia de género en sus trayectorias de vida (véase cuadro 1 ).

La mitad de las mujeres participantes había padecido violencia de tipo sexual. No siempre fue en el viaje de tránsito y, lastimosamente, para algunas fue tanto en el viaje como en su vida previa.

Siguiendo la invitación de Wimmer y Glick Schiller para evitar nacionalismos metodológicos en la investigación social (2002)-dar por hecho ciertas ideas dentro de un Estado-nación-fue esencial reorientar lo que de inicio se daba por sentado para dicho proyecto: que la violencia sexual ocurría solamente en el tránsito. Emergió entonces un continuum de violencias, violencias silenciadas que se han interiorizado simbólicamente y que suelen ser fragmentadas en nuestras investigaciones.

Al hablar de los motivos de salida de sus países, como se muestra en el cuadro 1, muchas mujeres narraron historias de abuso sexual desde la infancia, usualmente por parte de algún familiar o cuidador primario. Asimismo, el maltrato infantil fue recurrente, ya fuera por parte de la madre, el padre, padrastro, madrastra o algún tío / tía o abuelo/abuela que estaba a cargo del cuidado de ellas en el caso de que los padres hubieran migrado a Estados Unidos. Además, en su mayoría, las entrevistadas tuvieron un acceso limitado a la educación, pues desde pequeñas fueron forzadas a trabajar en el campo o en el comercio informal.

Los casamientos a edad temprana (entre los $12 \mathrm{y}$ los 15 años) fueron comunes en las tramas recopiladas y, sin excepción, fueron seguidos de violencia de la pareja.

Se encontró que los cuerpos de las mujeres migrantes constituyen un territorio permanente de disputas y agresiones por parte de sujetos masculinizados de diversa índole.

Es importante destacar que son mujeres de origen centroamericano, en concreto del triángulo norte de Centroamérica (Nicaragua, Honduras, El Salvador y Guatemala), que provienen de contextos precarios de países en donde los conflictos internos de los ochenta nunca terminaron de resolverse. Aun cuando sus países ya no están en guerras formalmente declaradas o abiertas, viven las resacas de conflictos sociales no resueltos por la falta de programas posconflicto que permitieran transitar del escenario de guerra a un entorno de paz y desarrollo social sostenible. En medio de ello, los cuerpos de las mujeres siguen siendo un territorio de control tanto en Centroamérica como en las rutas migratorias y en los países donde se establecen, porque los grupos de sicarios que tienen el dominio del mercado migratorio en México también disputan sus cuerpos para ejercer su control y casi siempre están situados en amplias redes desde sus países de procedencia. Se trata, entonces, de una forma de ejercicio de poder que trasciende las fronteras entre países de la región Centroamérica-México-Estados Unidos (Barja, 2019).

Estas mujeres viven violencias en sus países de origen, en sus experiencias de viaje de tránsito y en

\section{Tipos de violencia identificada en mujeres migrantes indocumentadas}

\begin{tabular}{lcc}
\hline \multicolumn{1}{c}{ Tipo de violencia identificada } & $\begin{array}{c}\text { Experiencias } \\
\text { documentadas }\end{array}$ & 3 \\
\hline Abuso sexual en la infancia en el país de origen & 1 \\
Violencia sexual en la vida adulta en el país de origen & 2 \\
Violencia doméstica en México & 1 \\
Violencia de pareja en el tránsito & 5 \\
Violencia sexual (tocamiento, acoso, relaciones sexuales de intercambio de favores, & \\
prostitución forzada, violación) en el tránsito por México & 2 \\
Violencia sexual en México como país de destino (acoso, violación) & 2 \\
Violencia física, psicológica y verbal en privación de la libertad (secuestro) & 11 \\
Violencia social y política (extorsiones, amenazas /atentados contra la vida) & \\
\hline
\end{tabular}

Fuente: Elaboración propia. 
los lugares donde se asientan. Por eso, estos nuevos lugares a menudo generan enclaves frágiles, al volverlas a exponer a ciclos de violencia constantes que, con reiteración, las expulsan a otro lugar.

Cuando en 2016 realizamos el segundo proyecto de diagnóstico de necesidades de poblaciones migrantes y refugiadas en la ciudad se organizaron grupos focales con mujeres en distintas casas de migrantes de la Ciudad de México y área metropolitana. Fue relevante toparse con la misma tendencia de violencias perpetradas hacia mujeres heterosexuales y mujeres trans tanto en los países de procedencia como en su experiencia de tránsito. Los siguientes testimonios constituyen ejemplos de las topologías de la experiencia vivida en el viaje de tránsito. Una experiencia de violencia que se produce a través del ultraje del cuerpo de un grupo concreto de mujeres: las jóvenes, pobres y morenas.

Estuvimos a punto de que nos violaran, nos desnudaron para buscar dinero en nuestras partes. Estuvimos con un psicólogo cuatro meses, sí nos ayudó bastante pero a uno nunca se le pasa, es mentira que esto un día se le pase.

Mi hija hasta la fecha me dice "Mami ¿verdad que ya no nos van a salir esos hombres? ¿por qué no nos regresamos a casa?" Hasta la fecha se come las uñas y se saca sangre de sus uñas.

En las zonas de tránsito, que por lo general son tierra de nadie, el cuerpo feminizado está expuesto y se convierte en un territorio que puede ser tomado a la fuerza por agresores de organizaciones ilegales y de organismos de seguridad estatales como policías y militares, o civiles con quienes conviven diariamente, como relataron algunas personas sobrevivientes de violencia sexual en el país.

Recurro al cuerpo feminizado para dar cuenta de los cuerpos que al margen de su constitución biológica y anatómica son construidos estéticamente como no-masculinos.

En los dos testimonios expuestos, las experiencias son coincidentes para una mujer trans y una heterosexual respectivamente. En ambos casos el cuerpo femenino constituye un instrumento de control ejemplar pues (a diferencia de otras mujeres en una posición económica más privilegiada o de hombres que provienen de los mismos ambientes precarios que forzaron la migración), generan una experiencia distinta, que produce la forma más extrema de sometimiento: ejercer el control territorial a través del control del cuerpo de ellas.

Estas formas de control territorial escritas sobre el cuerpo femenino pueden ser expresiones de violencia abiertamente sexual (violación, un tocamiento, desnudarlas, exhibirlas ante sus compañeros migrantes, ante policías, sicarios o delincuentes locales), o manifestaciones en códigos más sutiles, que de igual manera invisten manifestaciones de violencia sexual. Por ejemplo, para las mujeres que se instalan en la Ciudad de México por temporadas variables, el cuerpo femenino y la sexualidad son vistos por la sociedad de acogida como un espacio público al servicio de otros, que se asocia incluso con estereotipos nacionales vinculados a la hipersexualización del cuerpo.

Como ejemplifican los siguientes extractos de la experiencia de mujeres que participaron en grupos focales, el cuerpo femenino es un territorio abierto para las miradas y comentarios lascivos de hombres en las calles, situación que no se asocia exclusivamente a la condición extranjera sino a la transversalidad de género para mujeres nacionales y no nacionales. Algunas relataron acoso y violencia sexual en las calles de la Ciudad de México.

Una vez me dijo un señor "Mira, si tienes tan bonito cuerpo ¿por qué no lo vendes y te pones en una esquina ahí, y me dejas a tus niños aquí? Yo te los cuido, ¿por qué no lo vendiste en tu país para sobrevivir en vez de venir aquí?”.

Acá también me pasó aquí en Hidalgo, en la estación del Metro, en el parque. Íbamos con otra mujer que estaba aquí y vemos a una señora que estaba ahí, y me dice, “¿Cómo vienen ustedes a pedir aquí? Dios para eso les dio las nalgas, mire allá, ¿ve aquellos hombres? Con doscientos pesos que les den y ustedes dejen de andar pidiendo. Si nosotras que estamos aquí somos prostitutas, no digamos ustedes".

Por su condición de extranjeras jóvenes, de clase social baja, su cuerpo es representado en el imaginario de los otros como un objeto de trabajo. En las narraciones, ellas hablan de encuentros con hombres y mujeres de México que, al reconocerlas como extranjeras provenientes de países pobres, piensan que están aquí porque se dedican al trabajo sexual, o que deberían dedicarse a ello en vez de pedir ayuda en las calles.

Muchas de las entrevistadas tienen historias de violencia previas, algunas han sido expulsadas de sus países a causa de la falta de protección de sus gobiernos para salvaguardar su vida. Esta condición se exacerba cuando se trata de una mujer transgénero o transexual:

Fui amenazada por unos soldados que me querían matar adentro de mi casa y me violaron, robaron mi dinero, mis cosas; tuve que venirme huyendo porque yo quería 
mi vida, había hecho tanto, hasta uno de mis sueños, trabajar en un canal de televisión ejerciendo lo que a mí me gustaba a pesar de toda discriminación.

A nosotras se nos obliga, aunque tengamos la preparación académica, a la prostitución, la sociedad te está jalando por un lado. No puedes traer ni el cabello largo, no puedes vestirte fashion, porque si eres así en mi país es como un pecado, lo miran como lo peor, y donde vayas a aplicar a una empresa así, bye, no puedes ingresar.

Ser trans todavía en Latinoamérica es bastante difícil, siento yo, y México es igual, aunque quiera decir lo que quiera, en México la discriminación, las mujeres son súper transfóbicas, cuando vas en el Metro se siente mucho la pesadez.

Una vez salí con una chica trans, salimos a la disco, entonces nos tocaba caminar demasiado para llegar a la casa donde yo me iba a quedar, íbamos solas en una calle sola. Entonces pasa un carro, era uno de los sicarios que había por donde yo vivo, vino y dijo que nos montáramos y yo lo conocía a él por que él enamoraba a una prima mía, pero yo nunca pensé que él fuera a hacer algo más y nos obligó a que nos montáramos a su carro y nos llevó hasta una casa baldía, a mí me dejó encerrada en el carro mientras sacó a mi otra amiga con la pistola y la metió a la casa baldía y ya después salió y me dijo que seguía yo.

Estas expresiones de violencia para las mujeres transgénero o transexuales han generado que un número creciente llegue a México a solicitar ser reconocidas como refugiadas. Una vez que cuentan con la protección internacional la problemática toma otros matices, pero no cesa:

Algunas veces somos perseguidas, hay mucho acoso, demasiado acoso sexual aquí es ¡uff! Increíble, cuando ves que un hombre te sigue, te sigue y te sigue hasta que le quieras dar algo y si no quieres estar con él jay no, qué horror!

Hay mucha gente que no está de acuerdo con la diversidad, hay muchísima discriminación y es ilógico no mencionarlo, también hay mucha violencia, eso no se puede dejar de olvidar, hay que mencionarlo porque siguen apareciendo muertas.

Rita Segato (2013) propone que estas formas de violencia hacia las mujeres son las nuevas modalidades de depredación hacia los cuerpos femeninos, modos que se instalan en sociedades latinoamerica- nas que hoy por hoy se distinguen por vivir guerras no formales entre Estados.

El trabajo de campo mostró con recurrencia que estas guerras no formales son turbias, porque los perpetradores son de carácter distinto. Un pandillero, que a la vez es familiar o amigo de la infancia, o un policía que está vinculado a las redes de tráfico y de trata en Honduras o El Salvador, o la pareja o un funcionario, y se perpetúa una cadena de maltrato que produce expulsiones masivas de mujeres, pese a que ellas sepan del peligro en los caminos. Todas las mujeres que compartieron su experiencia sabían que habría igual o más violencia al venir a México. La simple posibilidad de encontrar un resquicio donde sortear o librar estas violencias hace que para ellas tenga sentido salir de sus países:

¿A qué me quedaba allá? ¿A que me mataran? Al menos aquí he pasado cosas terribles, pero le puedo decir que no peor que allá, y aquí quizá encuentro una nueva oportunidad.

Esta afirmación de una de las participantes de uno de los grupos focales fue secundada por las otras 12 que estaban en la charla. Y, aunque el panorama se ve bastante desolador, las personas en movilidad en el mundo hoy han retado la idea de pertenencia a un Estado-nación desprendiéndose del territorio nacional. La gestión política de sus cuerpos ocurre dentro de los territorios donde nacieron y se ejerce mediante la violencia hacia sus cuerpos. Para estas mujeres, salir del país donde se radicaba representa un pequeño acto de des-sujeción ante las estrategias de control que mantienen el orden de opresión hacia ellas.

Todas las personas construidas en código femenino, en su calidad de no-hombres enfrentan las mismas problemáticas asociadas a los riesgos sexuales, a la creencia del mundo masculino de que los cuerpos feminizados son propiedad de ellos. Los tocamientos, las violaciones, los feminicidios, los crímenes de odio $\mathrm{y}$ las distintas formas de violencia sexual no son exclusivos de las poblaciones migrantes, pero sí se potencializan en los ambientes clandestinos en que ocurre la migración irregular, y no en todos los casos cesan, incluso si las personas han logrado regularizar su situación migratoria. Además, son delitos menos propensos a ser perseguidos y castigados.

En el lugar de la experiencia, anunciado por De Lauretis, encontramos esta construcción diferencial de las relaciones sociales de sujeción que distan considerablemente de la experiencia del viaje de tránsito de hombres migrantes que viven violencia y son sometidos mediante otros mecanismos de control. 


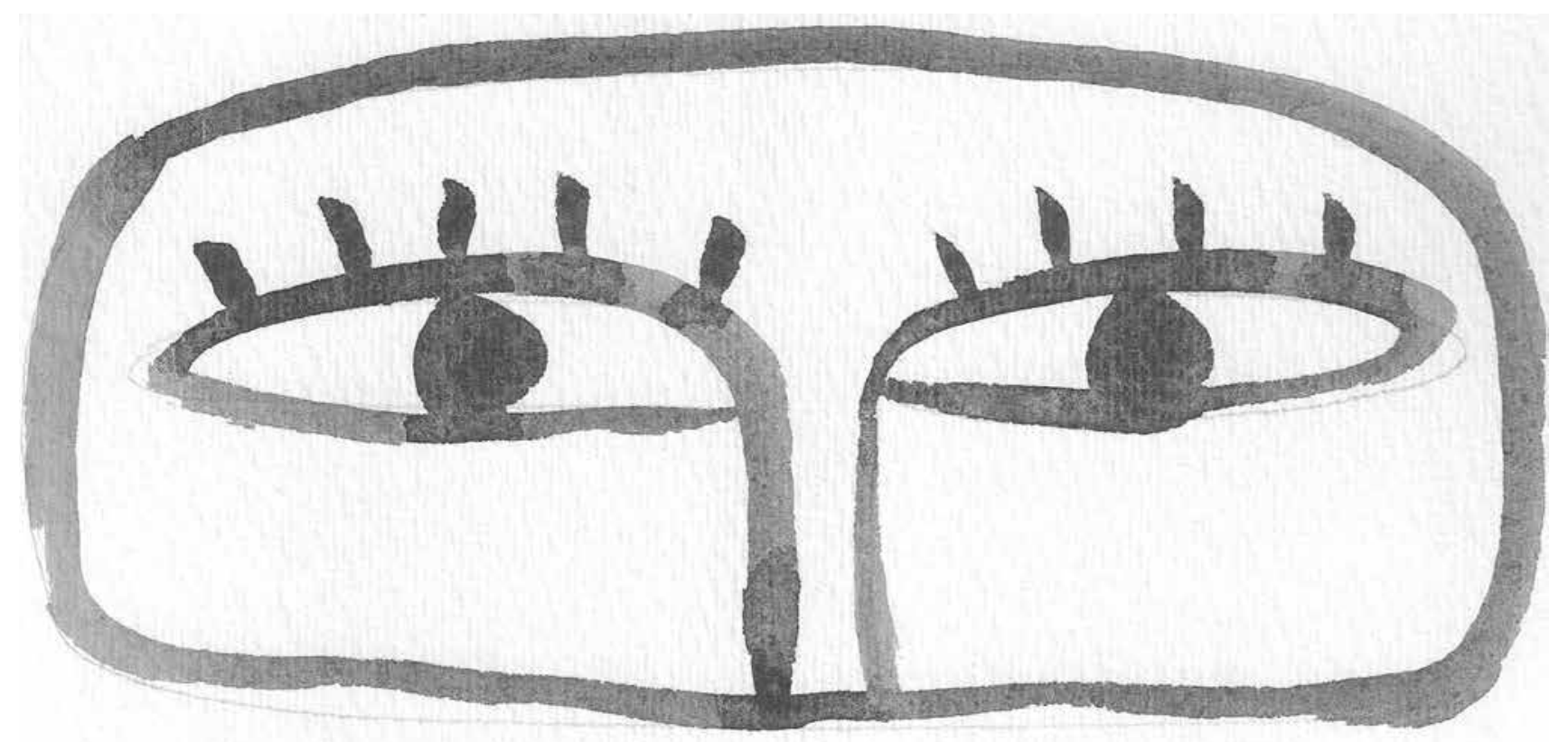

Para las mujeres heterosexuales o transexuales el cuerpo feminizado es el lienzo en el que se inscribe la pedagogía de la crueldad que tiene la intención de adoctrinar y mostrar control. Más el cuerpo es también territorio de disputa a los órdenes sociales hegemónicos, como ocurre al irrumpir en el orden heteronormativo de identidad sexogenérica. Las mujeres migrantes centroamericanas indocumentadas corporizan la posición de mayor desventaja dentro de la cadena de abuso y opresión que viven quienes migran en condiciones precarias, pero su capacidad de reconstitución corporal y subjetiva es el legado histórico que materializa la lucha que desde niñas tienen que dar para poder conservar la vida.

En la experiencia vivida propuesta por hooks encontramos la materialización de la construcción genérica diferencial que viven las mujeres en función de otras categorías como sexo, raza, etnia o clase social. Y es también en la experiencia vivida que hallamos las pistas de las estrategias que permiten des-sujetarse de los órdenes de control hegemónico que las han oprimido desde la vida temprana.

Algunas aprendieron a vivir solas desde la infancia porque la violencia doméstica o sexual las expulsó de casa, y fue esa movilidad forzada en la microescala de su localidad la que las produjo con el potencial de saber vivir vidas itinerantes, hoy aquí, mañana allá. Con frecuencia mencionaron que no tenían miedo de continuar porque estaban habituadas a adaptarse a los cambios si eso podía darles una mejor oportuni- dad de vida. Contrario a las narrativas hegemónicas, estas mujeres se caracterizan por tener independencia económica, aun cuando en muchos casos cumplir un rol familiar asociado a la maternidad les limita de otras formas de autonomía.

Resulta importante resaltar que, de acuerdo con sus relatos, para varias de ellas esa experiencia de autonomía económica se gestó a partir de la experiencia de viaje de tránsito por haberse separado de sus parejas o porque el contexto necesitaba que ellas tuvieran sus propios recursos para sobrevivir. Un ejemplo muy claro es que las mujeres en tránsito rara vez charolean, ${ }^{4}$ a diferencia de los hombres. Ellas están en las calles en los mismos cruceros que los hombres migrantes, pero recurren a la venta de dulces o al trabajo en alguna cocina, también hacen servicios de limpieza o de cuidados en los espacios que nos rodean.

Vemos así que migran porque incluso frente a nuevas expresiones de violencia que viven en México su identidad ha sido constituida de forma maleable para reconstituirse permanentemente. El miedo, aunque esté presente en su vida, no restringe sus decisiones ni sus aspiraciones por tener una vida más digna o lejana a la violencia. La vida itinerante ha sido una parte central en su constitución subjetiva, y la aspiración a encontrar espacios libres de estas violencias históricas es uno de los sostenes principales para decidir tomar el riesgo. Hubo mujeres que no obstante haber padecido varios episodios de violencia comentaron que lo volverían a hacer sólo por la posibilidad

\footnotetext{
4 Expresión coloquial utilizada en el argot migrante para describir la práctica de salir a la calle a pedir dinero para seguir el camino.
} 
de haber sacado a sus hijas del riesgo latente del control de pandillas en sus países. Romper la cadena de violencia de forma histórica y transgeneracional, a través de liberar a sus hijas de esos riesgos, dota de sentido la experiencia de la migración en medio de la violencia.

Otras mujeres también han aprendido a jugar estratégicamente con su propia identidad, en ocasiones se asumen como migrantes si esa posición las coloca en la posibilidad de modificar el contexto hostil en que están, aunque sea en lo inmediato (por ejemplo cuando la vivencia migrante les permite acceder a espacios de alojamiento que los hombres no tendrán o a documentos migratorios que les darán la tranquilidad de vivir en México de manera temporal sin estar expuestas al acoso constante).

Es así que en la experiencia vivida se ejecutan acciones estratégicas para evitar el sometimiento que la violencia consustancial de la migración, en código de género, busca reproducir sistemáticamente al grado de aniquilarlas materialmente. Esta lucha ocurre incluso en pequeñas acciones cotidianas.

Hay mujeres trans que declararon decidir de manera estratégica la estética de su cuerpo en código de masculinidad para rearticular las relaciones de poder en los microespacios sociales y disputar la posibilidad de no verse controladas en ciertos escenarios.

\section{Algunos dilemas del trabajo de campo en contextos de violencia}

Por último, expondré algunos de los dilemas que el tema de la violencia representa para quien investiga a la luz de las particularidades de la antropología. Un primer punto es que la violencia no está objetivada y es parte de las relaciones en las que quien investiga construye su trabajo analítico y de campo.

Esta sección la planteo desde mi propio enfoque situado. Mujer citadina, psicóloga y antropóloga, con trabajo previo en el tema que aquí se investiga desde el ámbito de las organizaciones de la sociedad civil.

Realizar trabajo de campo en un terreno que ya conocía supuso ventajas y desventajas. Por un lado, tuve un acceso relativamente sencillo a las casas de migrantes donde recogí los datos etnográficos. Insertarme en las tareas de estos espacios fue fácil en la medida que ya sabía como funcionaban algunas de sus prácticas. Pero fue un arma de doble filo el pisar el terreno de manera "práctica", por no contar con los protocolos adecuados de quien investiga en un terreno donde la violencia está latente. La realidad nos invita siempre a replantear el camino.
Subir a un tren que se puso en marcha inesperadamente sin saber si podría bajar pronto o no; cuidar a un par de niñas que habían sido llevadas a un albergue por un coyote que supuestamente las reuniría con sus padres en Estados Unidos y, en medio de amenazas al albergue, recontactar a la familia en el Salvador para reunificarlas sin ponerlas en riesgo con un "desconocido"; transitar por las calles de la Ciudad de México donde se ubica la casa de mujeres migrantes y recibir llamadas para entrar tan veloz como pudiera a la casa y no salir hasta que los policías de la zona dieran aviso, porque algún operativo ponía en riesgo a las migrantes; o hacer un vínculo empático con una migrante exmilitar que, en su afán de colaborar con mi investigación, me propuso recurrir a acciones de espionaje para "nutrir mi investigación" con datos fuera de lo común, son algunos de los escenarios que ejemplifican el tipo de dilemas situacionales que enfrenté en el tránsito por este trabajo. ¿Errores metodológicos? ¿Errores de protocolo? ¿Dónde está el límite entre nuestra labor antropológica y el ambiente de inseguridad del cual de alguna manera también somos partícipes? Estos trozos de realidad de los escenarios de violencia en los que realizamos nuestra investigación nos interpelan y nos mueven a pensar en unas condiciones mínimas para llevar a cabo un trabajo de campo que, en la vehemencia de hacer ciencia, no sobreexponga nuestra integridad ni arriesgue a las personas que participan y son el centro de nuestra investigación.

La propuesta a partir de esta experiencia es un quehacer de antropología colectiva. La seguridad o inseguridad rebasan el ámbito de las decisiones personales de quien investiga. Los posibles escenarios o construcción de redes de soporte con el apoyo de nuestras instituciones académicas son un espacio de arranque. Los espacios postrabajo de campo entre quienes hacemos antropología de la violencia hoy son una práctica que debe echarse a andar en paralelo al desarrollo de nuestras investigaciones. Es menester contar con protocolos mínimos para medir riesgos potenciales y riesgos inminentes. Estos protocolos deben establecerse con la asesoría de las personas que diariamente están inmersas en lo que nosotros definimos como nuestro terreno de campo. Es necesario definir rutas claras para realizar acciones de emergencia en caso de estar expuestas o de que nuestras sujetas de estudio lo estén. Este tema va más allá de las cartas de afiliación institucional que habitualmente nos abren las puertas al terreno de la investigación. Los espacios académicos en los que se trabaja violencia deben estar abiertos a la discusión de la dimensión afectiva de quien estudia violencia y vuelve del trabajo de campo. 


\section{Reflexiones finales, ¿por qué si hay tanta violencia en el camino ellas siguen migrando?}

A partir del caso expuesto considero que, en los temas que involucran problemáticas sociales que nos trastocan, la antropología tiene un compromiso ineludible, acercarse en profundidad a estos temas a partir de su metodología característica: introducirse a la cotidianidad de sus interlocutores para entender las prácticas y sentidos que explican el mundo social.

Más todavía, en cuanto al tema de la violencia, es necesario generar discusiones sobre las maneras y los contenidos de estas formas prácticas de hacer antropología. Del modo en que producimos etnografías al límite, como las llama Francisco Ferrándiz (2011).

Ello implica el ejercicio reflexivo del acercamiento antropológico que proponemos para el terreno de campo, así como del posicionamiento que asumimos en las relaciones que construimos ahí, y las formas mismas en que la violencia nos construye como investigadores. Pensarnos en referencia a la propia relación de privilegio respecto de quienes participan de nuestra investigación es una tarea también necesaria que hay que explicitar en nuestros respectivos trabajos. Se trata de prácticas esenciales en la antropología. Omitir estas reflexiones tiene un efecto político en la producción científica a la que pretendemos contribuir: nos desanclamos del ejercicio de vinculación con el otro que distingue históricamente a la antropología.

Ahora, para concluir, me remitiré a la pregunta inicial: por qué si hay tanta violencia ellas siguen migrando.

A la luz de la contribución de una ciencia antropológica lo suficientemente relevante para incidir en el debate público e instrumentalizar la práctica antropológica en cuanto a su papel político para la mejora de las sociedades existentes (Krotz, 2018), es indispensable proponer acciones que atiendan las problemáticas de la experiencia vivida de las mujeres migrantes.

La construcción del cuerpo feminizado para muchas mujeres heterosexuales y transgénero o transexuales es sistemáticamente violenta en sus países de origen y fuera de ellos. Inmersas en un continuum de violencias histórico y transnacional, ellas viven reiteradamente distintas modalidades de control que operan a través de sus cuerpos y responden a lógicas de organización social, no exclusivas de las migraciones.

Pese a saberse potencialmente expuestas a nuevas expresiones de violencia si realizan un movimiento transfronterizo dentro de los circuitos de la ilegalidad, seguir migrando es comprensible, no desde nuestra lógica de espectadores, sino desde la lógica de sentido de mujeres que han estado de manera permanente expuestas a la violencia. La labor antropológica invita a mirar estas otras tramas de sentido que ante los ojos ajenos se reducen a expresiones coloquiales del tipo: "si hay tanta violencia en el camino y saben que incluso las pueden violar, ¡cómo es que se arriesgan a venir por estos caminos!", para adentrarnos en el sentido que para cada una de ellas adquiere la movilidad transfronteriza. Es aquí donde el concepto de experiencia vivida puede ser central para entender la producción de subjetividades y materialidades diversas investidas de un discurso hegemónico llamado migración en tránsito, que pareciera aludir a experiencias similares de un grupo homogéneo: los migrantes.

La "migrante en tránsito" que arribó al país en una caravana, en principio hacia Estados Unidos, que lleva más de seis meses trabajando en el comercio informal, ha tenido un hijo en México y está buscando opciones de regularización migratoria, ¿será migrante en tránsito desde su propia autoconcepción?

La "inmigrante" que llegó como refugiada al país y ha regularizado su estancia migratoria para producir una nueva movilidad para reunificarse con su familia en Canadá será ¿inmigrante?, ¿migrante en tránsito?

Quien ha entrado a Estados Unidos y ha sido deportada tres veces a su país y se encuentra nuevamente en territorio mexicano acumulando experiencias de vida, trabajo y relaciones sociales en el lapso de los últimos cuatro años, ¿podrá ser considerada migrante de retorno dentro de México, aunque el retorno suele estar asociado al país de origen y no al de tránsito?

Emigración, inmigración, migración de retorno o en tránsito más que categorías analíticas resultan ser constructos que sirven en la elaboración de políticas migratorias, en particular cuando los Estados necesitan producir nuevos órdenes de control fronterizo. Estos constructos se vuelven inaprensibles en la experiencia vivida. De ahí que no se pueda dar una respuesta contundente a las preguntas formuladas en los párrafos anteriores, porque estamos ante una realidad social mucho más compleja.

Para la investigación reseñada, propongo como alternativa considerar la movilidad humana en cuanto espacio dinámico de la experiencia vivida que incorpora las complejidades descritas. Dentro de tales movilidades habrá que tener en cuenta que la violencia no es una caracterización exclusiva del viaje migratorio clandestino. Pensar que es así es reproducir la gran narrativa mediática del espectáculo de la migración (cf. Barja, 2019). A la luz de los datos ofrecidos, la construcción genérica de la violencia sobrepasa el tema de las migraciones. La violencia durante el viaje de tránsito es una pieza más en el continuum de violencias 
que viven las mujeres. Cambian los actores y los espacios donde la violencia se reproduce, pero conserva el común denominador de sometimiento del cuerpo a través de la sexualidad femenina que ingresa en una economía de intercambio masculina. Establecer límites claros entre el objetivo de ese intercambio en el contexto de procedencia y en el del viaje de tránsito es poco viable, ya que, como se escribió en los apartados anteriores, aun en el ámbito de la violencia familiar, los perpetradores pueden estar vinculados con pandillas y grupos criminales transnacionales que son los mismos que operan y controlan las rutas de tránsito.

En términos de políticas públicas, atender la violencia sexual vivida por mujeres migrantes "en tránsito por México" requiere una mirada más amplia y capaz de interesarse en el análisis de las violencias de vida sin importar el territorio donde ocurran ni la constitución biológica del cuerpo humano. Con los casos presentados he buscado poner énfasis en las formas de producción de violencia ejercidas sobre cualquier cuerpo que no cumple el estándar masculino.

Esta mirada invita a construir protocolos de atención con sentido en las instituciones judiciales y de salud pública encargadas de atender a mujeres que con frecuencia son vistas como víctimas con poca posibilidad de agencia para la reconstitución de sus vidas. Transformar estas miradas puede ser una vía de acción para la inclusión, en la medida en que son tomadas en cuenta desde su propia lógica de vida, entendiendo los sucesos violentos que han enfrentado y reconociendo las acciones reparadoras que pueden tener significado para ellas.

\section{Fuentes}

Amnistía InTERnACIONAL

2010 Víctimas invisibles: Migrantes en movimiento en México, Amnesty International Publications, Madrid, $42 \mathrm{pp}$.

BARJA, JOSELIN

2019 “En este mar de absurdas persecuciones'. Presencias migrantes centroamericanas en contextos de violencia", tesis de doctorado, Universidad Autónoma Metropolitana, Unidad Iztapalapa, 389 pp.

BarJa, Joselin y Vladimir Tlali

2017 "iLo que no tenemos y necesitamos se llama inclusión!" Diagnóstico interseccional sobre la situación y necesidades de mujeres; niñas, niños $y$ adolescentes; y personas LGBTTTI migrantes y sujetas de protección internacional en la Ciu-

Besserer, Federico dad de México, Sin Fronteras, México, 87 pp.

2004 Topografías transnacionales. Hacia una geografía de la vida transnacional, Universidad Autónoma Metropolitana/Plaza y Valdés, México, $125 \mathrm{pp}$.
BLAIR, ELSA

2002 "La complacencia en el exceso", en JOVENes, Revista de Estudios sobre Juventud, vol. 6, núm. 16, pp. 58-76.

Castro, Yerko

2017 "Etnografías de la violencia. Dilemas para hacer y pensar las etnografías en zonas de guerra, violencia y conflicto", en Yerko Castro y Adèle Blazquez (coords.), Micropolíticas de la violencia. Reflexiones sobre el trabajo de campo en contextos de guerra, conflicto y violencia. Cuaderno núm. 5, LMI Meso, México, pp. 57-67.

De la O, María Eugenia

y Manuela Camus

2018 "Mujeres centroamericanas en tránsito irregular por México: dinámicas y experiencias de vida", en Rosana Baeniger y Alejandro Canales (coords.), Migraciones fronterizas, Universidade Estadual de Campinas, Campinas, pp. 202-216.

DIARIO IMAGEN

2018 "Caravana migrante y la ruta del infierno". 23 de octubre <https: / /www.diarioimagen.net/ $? \mathrm{p}=400309>$ [24 de noviembre de 2018].

Estévez, Ariadna

2018 "El dispositivo necropolítico de producción y administración de la migración forzada en la frontera Estados Unidos-México", en Estudios Fronterizos, vol. 19 <http://dx.doi.org/10. $21670 /$ ref. $1810010>$

FERrándiz, Francisco

2011 Etnografías contemporáneas. Anclajes, métodos y claves para el futuro, Anthropos, Madrid, $271 \mathrm{pp}$.

García Aguilar, María del Carmen

y Daniel Villafuerte Solís

2014 Migración, derechos humanos y desarrollo. Aproximaciones desde el Sur de México y Centroamérica, Universidad de Ciencias y Artes de Chiapas / Juan Pablo Editor, Londres, 423 pp.

Goldberg, Eleanor

2014 "El 80\% de las niñas y mujeres centroamericanas son violadas al cruzar hacia EE.UU”, trad. de María Guardado, 16 de septiembre The Huffington Post <https://www.sinembargo. $\mathrm{mx} / 16-09-2014 / 1119157>$ [Versión original en Huffpost Impact: <https: / /www.huffpost. com / entry/central-america-migrants rape_n_5806972?ncid=fcbklnkushpmg 00000063\&guccounter $=1$ \&guce_referrer $=$ a HRO c DovL 3 ZvY 2 Vz MTA 1 L n J z c $21 \mathrm{u}$ Zy5jb20vY2hhbiOyNTE2Mzk0OC9hbGxfc DE2MC5odG1s\&guce_referrer_sig=AQAAA JZArm3hEtXPxoJokemJ9fr5-js7wuoaE0 n p seh - rL 9 zjpE - y r K 7 y Md - B tiNXB 2 n WrcVH-59wPbErkmR6DaIHcLeU4-JHyns GU 11 v9OrZ4Zvhymb-xO EvEQRrxJsvXU rnO5HQ2s 2XBcgYNmhiM $18 \mathrm{kx} 6 \mathrm{mfJiB} 2 \mathrm{CI}$ kTO6y7KuXEZV> [ 18 de septiembre de 2018].

Gómez-Johnson, CRistina

2015 "De la migración económica a la migración forzada por el incremento de la violencia en El Salvador y México", en Estudios Políticos, núm. 47, pp. 199-220.

HOOKS, BELL

2004 "Mujeres negras. Dar forma a la teoría feminista", en bell hooks, Avtar Brah, Chela Sandoval y Gloria Anzaldúa, Otras inapropiables. 
Feminismos desde las fronteras. Traficantes de sueños, Madrid, pp. 33-50.

Knippen, José, Clay Boggs

y Maureen Meyer

2015 Un camino incierto. Justicia para delitos y violaciones a los derechos humanos contra personas migrantes y refugiadas en México, The Washington Office of Latin America/Fundar Centro de Análisis e Investigación/Casa del Migrante Saltillo/Un Mundo una Nación/ Centro Comunitario de Atención al Migrante y Necesitado/Centro de Recursos para Migrantes/La 72, Hogar Refugio para Migrantes/ Hermanos en el Camino/Kino Border Initiative, México.

KROTZ, Esteban

2018 "Antropología: conocimiento y política", en Encartes Antropológicos, vol. 1, núm. 1, marzoagosto, pp. 67-83.

Lauretis, Teresa de

1984 Alicia ya no. Feminismo, semiótica, cine, Cátedra, Madrid, 295 pp.

LEFEBVRE, HENRI

2013 La producción del espacio, Capitán Swing Libros, Madrid, 451 pp.

Marcus, George

2001 "Etnografía en/del sistema mundo. El surgimiento de la etnografía multilocal", en Alteridades, año 11, núm. 22, pp. 111-127.

Mendoza, Jorge

2004 "Las formas del recuerdo. La memoria narrativa", en Athenea Digital, núm. 6, otoño <https: / / ddd.uab.cat/pub/athdig/ 15788946n6/ 15788946n6al1.pdf> [15 de noviembre de 2018].

NTR PERIOdismo CRítico

2011 "Tenosique, ruta de terror para migrantes". 5 de febrero <http://ntrzacatecas.com/2011/ 02 / 05 / tenosique-ruta-de-terror-paramigrantes / > [18 de septiembre de 2018].

Organización Mundial de la SAlud

2011 "Violencia contra la mujer: violencia de pareja y violencia sexual contra la mujer", Nota des- criptiva núm. 239, Organización Mundial de la Salud, Ginebra.

PARIS-Pombo, Dolores

2016 "Trayectos peligrosos: inseguridad y movilidad humana en México", en Papeles de Población, núm. 90, pp. 145-172.

Pintin-Perez, Margarita,

Martha Luz RoJas Wiesner

Y RUPALEEM BHUYAN

2018 "The Symbolic Violence of Tolerance Zones: Constructing the Spatial Marginalization of Female Central American Migrant Sex Workers in Mexico", en Women's Studies International Forum, vol. 68, mayo-junio, pp 75-84.

REDODEM

2015 Migrantes invisibles, violencia tangible. Informe 2014, Red de Documentación de las Orga-

Segato, Rita nizaciones Defensoras de Migrantes, México.

2013 Las nuevas formas de la guerra y el cuerpo de las mujeres, Tinta Limón, México, $121 \mathrm{pp}$.

Varela Huerta, Amarela

2017 "Las masacres de migrantes en San Fernando y Cadereyta: dos ejemplos de gubernamentalidad necropolítica”, en Iconos. Revista de Ciencias Sociales, núm. 58, pp. 131-149<https: / / doi.org/10.17141/iconos.58.2017.2486>.

WILLERS, SUSANA

2018 "Migration and Reproductive Strategies of Central American Women in Transit through Mexico", en Journal of Family Studies, vol. 4, núm. 1, pp. 59-75.

WimMER, ANDREAS

Y NiNA Glick SCHILlER

2002 "Methodological Nationalism and Beyond: Nation-State Building, Migration and the Social Sciences", en Global Networks, vol. 2, núm. 4, pp. 301-334.

Zavaleta, NoÉ

2013 "El infierno de los migrantes", en Proceso, 12 de junio <https: / / www.proceso.com.mx/ 344659/el-infierno-de-los-migrantes> [ 10 de septiembre de 2018]. 